4. The twelve elliptic functions considered by Glaisher and other writers are shown in Professor Bell's paper to arise as degenerate forms from a set of sixteen doubly periodic functions of the second kind which may be generated by the operations of a certain group from any one of them. The same functions give also, by differentiation, transformations of the second order, etc., all of the doubly periodic functions of the third kind considered by Hermite, Biehler, Appell, and others, with many more. The paper will appear in the Messenger of Mathematics.

5. In connection with his recent determinations of the numbers of representations of integers as sums of 3,5,7, 9, 11, and 13 squares, (which will appear in the American Journal), Professor Bell remarked that incidentally the 3-square results yield the class number by finite processes only. In this paper new binary class number formulas and analogous results related to quinary and senary forms are derived.

6. The equation solved in Professor Bell's note is that for the square of the line element in the general non-euclidean time-space, which holds a central position in Einstein's theory of gravitation. The solution is not free from quadratures. It is shown that particular solutions of certain other equations in general dynamics may be similarly found. The note will appear in the Philosophical Magazine.

B. A. Bernstein, Secretary of the Section.

\title{
THE SUM OF THE FACE ANGLES OF CERTAIN POLYHEDRONS IN $n$-SPACE.
}

BY DR. HARRIS F. MACNEISH.

(Read before the American Mathematical Society December 30, 1919.)

A SIMPLE polyhedron in $n$-space is defined as a set of $r$ spaces ${ }_{n-i} C_{r-i}$ of which intersect in each $i$-space; $r>i$; $r=1,2, \cdots, n-1 ; i=0,1, \cdots, n-2$.

For a simple polyhedron $P^{n}$ in $n$-space we denote the sum of the angles of all the plane faces by $S_{p n}$, the number of 
vertices by $p_{0}{ }^{n}$, the number of line edges by $p_{1}{ }^{n}$, and the number of $r$-space faces by $p_{r}{ }^{n} ; r=2,3, \cdots, n-1$.

For a simple polyhedron in $n$-space we develop the following formula for the sum of the face angles:

$$
S_{p n}=\left[\frac{1}{3} n(n-1) p_{0}{ }^{n}-\frac{4}{n-2} p_{3}{ }^{n}\right] \pi .
$$

For space of three dimensions for any simple polyhedron $P^{3}$ we have the Euler formula

$$
S_{p 8}=\left(p_{0}^{3}-2\right) 2 \pi .
$$

In space of four dimensions we consider any simple polyhedron $P^{4}$. In any 3 -space face $A_{i}{ }^{3} ; S_{A_{i}{ }^{3}}=\left(a^{3}{ }_{0, i}-2\right) 2 \pi$. Taking the sum for all of the 3 -space faces of $P^{4}$, since in a simple polyhedron four 3 -space faces pass through each vertex, and two 3-space faces through each plane,

$$
2 S_{p^{4}}=\left(4 p_{0}{ }^{4}-2 p_{3}{ }^{4}\right) 2 \pi, \quad \text { or } \quad S_{p^{4}}=\left(2 p_{0}{ }^{4}-p_{3}{ }^{4}\right) 2 \pi .
$$

To obtain an induction proof we assume the generalization of this formula for space of $r$-dimensions, i.e.,

$$
S_{p r}=\left[\frac{1}{3} r(r-1) p_{0}{ }^{r}-\frac{4}{r-2} p_{3}^{r}\right] \pi .
$$

In $(r+1)$-space the sum of the plane face angles of each $r$-space face $P_{i}^{r}$ of a simple polyhedron $P^{r+1}$ is given by the above formula.

Taking the sum of the plane face angles for all the $r$-space faces of the polyhedron $P^{r+1}$, since $(a) r+1 r$-space faces pass through each vertex; $(b) r-2 r$-space faces pass through each 3-space and (c) $r-1 \quad r$-spaces pass through each plane, in a simple polyhedron in $(r+1)$-space

$$
S_{p r+1}=\left[\frac{1}{3} r(r+1) p_{0}{ }^{r+1}-\frac{4}{r-1} p^{{ }^{r+1}}\right] \pi,
$$

which completes the induction proof. 